\title{
Novel Investigative Preparation of Human Hair
}

\author{
Isabel N. Boona ${ }^{1}$, Robert E.A. Williams ${ }^{1}$, Daniel Huber ${ }^{1}$, Jennifer M. Marsh ${ }^{2}$, Marc Mamak $^{2}$, David W. \\ $\mathrm{McComb}^{1}$ \\ ${ }^{1}$ Center for Electron Microscopy and Analysis, The Ohio State University, Columbus, OH, USA \\ 2 The Procter and Gamble Company, Mason Business Center, 8700 Mason Montgomery Road, Mason, \\ $\mathrm{OH}, \mathrm{USA}$
}

Preparing hair samples for electron microscopy has been problematic for various reasons. Keratinized hair is densely packed and inherently dry with the proteins heavily cross-linked[1]. This eliminates the need for primary and secondary fixation. However, the inability to uniformly stain through the cuticle layers and throughout the central cortex has shown varying results[1]. The hair samples in this investigation were treated with an oxidative permanent colorant and washed in tap water containing low levels of copper $(\mathrm{Cu})$. The amount of $\mathrm{Cu}$ in the hair was confirmed using inductively coupled plasma optical emission spectroscopy (ICP-OES). In order to optimize and better understand the effect of sample preparation on maintaining the native hair structure as well as internal chemical composition, analytical electron microscopy (AEM) characterization was performed [2,3]. In particular, techniques such as S/TEM-HAADF imaging and Super-X XEDS were used to investigate the ultrastructure and chemistry of the cross sectional surface of several hair fibers.

For the ultramicrotome samples, hair fibers were embedded directly with 2-part epoxy and cured at room temperature. The fibers were sectioned using a Reichert-Jung Ultracut E microtome with Diatome Diamond knife. As thin sections were produced, they were floated onto a DI water bath, but removed as quickly as possible in order to minimize possible interactions of copper ions with water. The thin sections were picked up onto gold grids $(0.5 \mathrm{~mm}$ slot $)$ with formvar/carbon support film.

A dual-beam focused ion beam (FIB) methodology was developed to prepare consistently thin, crosssectional specimens of hair for subsequent analysis. S/TEM-HAADF imaging was used to determine the homogeneity of the cross sections as well as reveal any artifacts that were introduced because of either sample preparation method. Figure 1(a,b,c,d) illustrates the two samples prepared by ultramicrotome and FIB at different magnifications. A comparison between the results obtained from the ultramicrotome sample and the FIB sample preparation techniques will be discussed.

Following TEM sample preparation, either by ultramicrotome or FIB, a monochromated Titan ${ }^{\mathrm{TM}}$ 60-300 STEM equipped with a Super-X XEDS collection system was used to investigate both samples as shown in Figure 2(a,b,c,d,e,f). The ultramicrotome sample, shown in Figure 2(a,b,c), clearly reveals the damage that was incurred by the preparation method which is similar to damage that has been shown in a previous example on ivory [4]. A successful minimal-damage methodology using the FIB was previously investigated on an organic photovoltaic [5] and in this case a hair sample prepared using the FIB, shown in Figure 2(d,e,f), exhibits little to no damage from this method and has allowed for further investigation of the $\mathrm{Cu}$ content in human hair [2,3].

This work has resulted in unparalleled characterization of human hair and demonstrated that FIB in combination with Super-X XEDS supersedes the norm for ultramicrotomy biological sample preparation. S/TEM HAADF imaging clearly reveals the difference between the two sample preparation methods. 
References:

[1] Harland, D. P., et al. J. of Microscopy 243(2) (2011): 184-196.

[2] Marsh, J. M., et al. Int. J. of Cosmetic Sci. 37(5) (2015): 532-541.

[3] Marsh, J. M., et al. Int. J. of Cosmetic Sci. 36(1) (2014): 32-38

[4] Jantou, V., et al. Micron 40(4) (2009): 495-501.

[5] Gilchrist, J.B., et al. 24(41) (2014): 6473-6483.

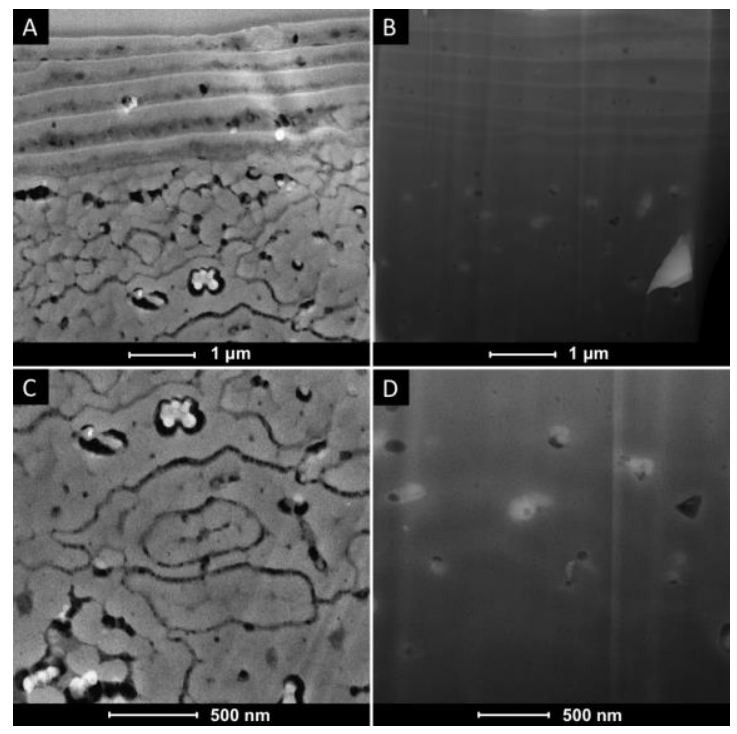

Figure 1. S/TEM-HAADF images of human hair. (a,c) Microtome sample (b,d) Focused Ion Beam (FIB) sample.
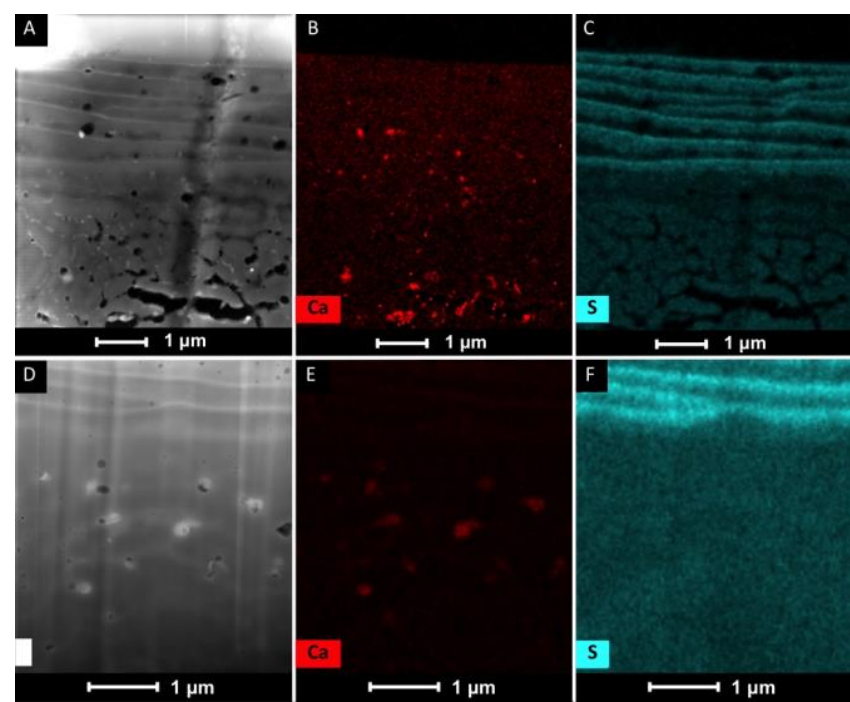

Figure 2. Data from the ultramicrotome $(A-C)$ and FIB $(D-F)$ sample of hair, A/D) STEMHAADF image, B/E) Calcium signal, C/F) Sulfur signal. The HAADF image of the ultramicrotome sample exhibits macrofibrils (MF) within the cortex of the hair surrounded by dark areas where the material is missing. In contrast, the FIB sample exhibits the MF surrounded by ribbons of material (lighter contrast) as well as white spots that closely correlate to the intermacrofibrillar matrix that is still intact. 